\title{
LA UNIVERSIDAD COMO EMPRESA NON PROFIT: EL CASO DE LA UNIVERSIDAD CATÓLICA SEDES SAPIENTIAE
}

Lic. Riccardo Spínelli

\section{Introducción}

La enseñanza es uno de los sectores en los cuales la presencia de operadores económicos non profit es tradicionalmente mayor: mucho más rara, sin embargo, es la experiencia de una Universidad non profit en un país en vía de desarrollo.

Esto es el caso de la Universidad Católica Sedes Sapientiae (UCSS), una Universidad «fronterizas ubicada en la periferia de Lima; un caso muy significativo, sobre todo considerando los grandes resultados conseguidos en estos primeros años de actividad.

Por lo tanto, puede ser interesante examinar sus caracteristicas estratégicas y operativas, para evaluar cientificamente y objetivamente la iniciativa y su eventual reproducibilidad en otros ámbitos de subdesarrollo.

Con esta finalidad se le aplicará el modelo de análisis de empresa conocido como «formula empresariab, elaborado por el estudioso italiano Vittorio Coda!

Esta modalidad de analisis deriva de la tendencia, en la moderna doctrina de la economia de empresa, hacia la progresiva consideración de las universidades como verdaderas empresas, suministradoras de servicios en el mercado de la ensenanza superior? ${ }^{2}$. Las lógicas empresariales de eficencias, eficacia y calidad tienen más y más relevancia en los órganos gestionales de los ateneos, públicos y

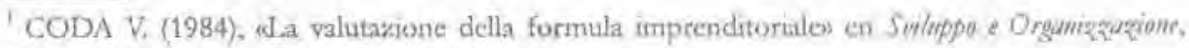
Núnero 82

"BACCARANI Cu (1999), “d,'Università tra cambiamento e conservazionen en Sinergie, Nürnero 48 enera-febrero 


\section{Riccardo Spine11i}

privados, también gracias a la drástica uretiradas del Estado; en muchos países occidentales, por ejemplo en Italia, se percibe, en efecto, una notable disminución de las ingerencias de las autoridades públicas centrales, con la atribución de poderes y competencias más y más amplios directamente a las universidades y, en vía indirecta, a los Departamentos.

En consecuencia, parece apropiado aplicar a una Universidad un típico modelo de análisis de empresa.

Se puede reconducir la formula empresarial, wel modelo de interacción con el ambiente a través del cual la empresa persigue su idea de éxito empresarial ${ }^{3}$ ' , a la resultante de las decisiones sobre el sistema de producto/servicio, el sistema competitivo ( $y$ en este caso también cooperativo), el sistema de los actores sociales, la propuesta proyectual y la estructura de la empresa: estos son los elementos que intentaremos evidenciar en relación a la UCSS, después de haber recorrido rapidamente su historia.

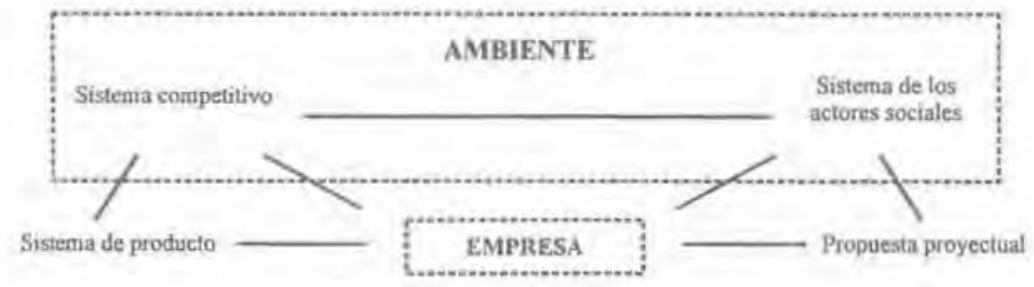

\section{La historia de la Universidad Católica Sedes Sapientiae}

La UCSS nace en diciembre del año 1999, por voluntad de Mons. Lino Panizza, obispo de Carabayllo, una de las cuatro Diócesis en las cuales está dividida la ciudad de Lima.

Una vez nombrado Obispo, Mons. Panizza, fraile capucino nacido en Italia, y en el Perú desde hace más de treinta años, hoy ciudadano peruano y desde siempre muy empeñado en el desarrollo social y cultural de Líma, percibe pronto el grave estado de deterioro de su territotio pastoral, la perifetia setentrional conocida como "Cono Nortes.

\footnotetext{
'CODA V (1988), wL'orientamento strategico dellimpresan en BLANCHI T, CODA V., MAZZA G., PAGANEI.LI O., PEIJ.ICELLI G.(eds.). Tratstoto di Econania Astenisile, Torino, UTET (T. d. A.)
} 


\section{La Universidad como empresa non profit: el caso de la Universidad Católica Sedes Sapientiae.}

Es una zona de reciente urbanización, objetivo de incesantes flujos migratorios desde diferentes regiones del pais $y$ hospeda, aproximadamente, dos millones de habitantes, de los cuales más de quinientos mil tienen edad inferior a los 15 años.

Como en otras áreas de la ciudad, las consecuencias de la superpoblación y del desorden urbano son evidentes para quien pase allí unos días: el tráfico es caótico, la polución atmosférica elevada, enteros barrios tienen enormes problemas ambientales e higiénicos, los servicios básicos están muy lejos de llegat a toda la población, la pobreza es evidente y de dramático impacto.

Con respecto al sistema educativo, vale la pena evidenciar unos datos estadísticos!

El nivel de analfabetismo es del $4 \%$, más alto que el $3,7 \%$ promedio de la ciudad de Lima. Asimismo, en el Cono Norte, en donde vive más o menos el $25 \%$ de la población total de Lima, se encuentran sólo el 19,91\% de las aulas. Es evidente la desproporción y la falta de estructutas, confirmada de los datos sobre el número de profesores, talleres, bibliotecas, etc. En todo caso, los problemas no son exclusivamente infraestrucuturales, sino de calidad de la enseñanza: se necesita, sin duda, elevar los estándares didácticos, desde las escuelas hasta las universidades.

La respuesta de Mons. Panizza a la petición de formación que le presentan todas las realidades sociales, políticas y económicas de la zona es muy clara: se empeña en la realización de centros de enserianza que llenen el vació debido a la falta de adecuadas estructuras públicas o privadas, para romper definitivamente la perversa espiral de pobreza e ignorancia que atenaza al Cono Norte.

Además de eso, sin embargo, se pide a la Iglesia una intervención a mucho más alto valor añadido, es decir, la formación de una nueva clase de docentes, preparados profesional y personalmente. El gran desafio es superar la carencia de una adecuada «cultura de la enseñanzas. Es el primer paso hacia la creación de un whombre nuevon, que sepa ser protagonista de su vida y contribuya al desarrollo de la realidad que lo rodea, atento, en la decadencia de los valores típica de la época contemporánea, a los fundamentos morales de la doctrina cristiana; se quiere canalizar la fuerte voluntad de rescate de los jóvenes del Cono Norte y exaltar sus potencialidades, ayudándolos en su crecimiento humano y profesional.

De aqui la idea de la creación de una nueva Universidad, para transformar y mejorar la sociedad empezando por los jóvenes. Una Universidad en una zona pobre de Lima, con la evidente intención de llegar a todos, de permitir acercarse a

\footnotetext{
'Imestigazön Socioeconomia sobre al Cono Norte, 2001, Lima, UCSS-CE,ASB
} 


\section{Riccardo Spinelli}

la cultura, incluso a quien hasta ahora ha sido excluido. Una Universidad seria y de alta calidad, que sea una válida alternativa a las costosas universidades privadas del centro de Lima y a las públicas, gratuitas pero de muy dificil acceso a causa de los severos exámenes de adinisión.

Las dificultades son imnumerables, ya sean financieras, debido a la necesidad de encontrar personas adecuadas para la formulación del proyecto y, luego, para la gestión del nuevo ateneo. Sin embargo, pesar de todo, lo que habia nacido como un «sueño" para Mons. Panizza se realiza.

Fundamental es la colaboración de una parroquia local, que ofrece el edificio, y de un grupo de profesores universitarios italianos activos desde hace unos años en el mundo académico limeño y próximos a un movimiento de inspiración católica que aceptan con entusiasmo hacerse cargo de la nueva Universidad. Se procede, luego, con la redacción del Proyecto Institucional, necesario para obtener la autorización de funcionamiento desde las autoridades competentes. Asi, el cia 26 de diciembre del año 1999, la UCSS es aprobada por el Concejo Nacional para la Autorización de Funcionamiento de Universidades (CONAFU) y, por lo tanto, puede empezar a obrar desde marzo del año 2000.

Hoy, la UCSS cuenta con dos facultades: Ciencias Económicas y Comerciales, y Ciencia de la Educación, y más de 1500 estudiantes, en ocho carreras. Puesto que actualmente funcionan los primeros seis ciclos, de los diez totales, se prevé que, una vez completados los ciclos, los estudiantes serán más de 3000 .

La UCSS es ya ana protagorista del panorama cultural, económico y social de Lima, también gracias a la actividad del Centro de Asesoria Empresarial (CEASE), del Centro Preuniversitario que organiza cursos en preparación del examen de admisión a la Universidad y del Centro de Idiomas.

\section{La aplicación del modelo de Coda}

Es ahora posible analizar el modus operandi de la UCSS y evaluar su contribución al proceso de desarrollo de Perú.

El modelo puede ser adaptado a un objeto atípico como una Universidad, además non profit, leyendo sus componentes desde un punto de vista parcialmente inusual.

¿Cual es, de hecho, la misión de la empresa aUniversidad Católica Sedes Sapientiaen? Por supuesto no es el beneficio, sino la formación y la activa contribación al desarrollo cultural, económico y social del entorno de referencia.

La mision que está đebajo de eso es la de una comunidad académica y científica que promueve la formación universitaria de los jóvenes, para hacer de ellos unos 


\section{La Universidad como empresa non profit: el caso de Ta Universidad Catбlica Sedes Sapientíae.}

profesionales responsables y unos hombres libres, preparados para enfrentarse a los problemas y a las necesidades de la vida.

Es un objetivo muy ambicioso, aun más en el contexto de la realidad peruana contemporánea. La UCSS intenta ser elemento propulsor de una srevolución culturals, actor de un "proceso de produccións que tenga como owtput individuos responsables y libres: responsables, hacia si mismos y hacia la sociedad; libres, porque instruidos serán capaces de defender sus derechos contra los abusos, de crecer personal y profesionalmente, de tutelar su identidad contra el neocolonialismo hecho por falsos mitos de progreso y de consumismo. $\mathrm{La}$ esperanza en esta visión es de lograr producir el cambio en el entorno circundante a través del rescate de cada individuo que, participando activamente a la vida social, revolucione los esquemas y guie un proceso de desarrollo centrado en el hombre.

¿Sin embargo, la UCSS logra consegur concretamente lo que se propone? Para contestar a esta pregunta es necesario, en primer lugar, sistematizar los elementos que constituyen su formula empresarial.

\subsection{El sistema de producto}

Con respecto al sistema de producto, es espontáneo preguntarse qué svendes una Universidad, en particular la UCSS, cuál será su uproductom. La respuesta no es fácil, puesto que las actividades de un ateneo son múltiples: además del tipico core business, hay muchas iniciativas colaterales, como la organización de eventos culturales, la asesoria a las empresas, la asistencia social hacia los estudiantes, la gestión de estructuras auxiliaries, por ejemplo, las bibliotecas, los centros deportivos o los labotatorios.

Intentando sintetizar lo más posible, se puede afirmar que la UCSS «venden un compromiso: compromiso en contribuir activamente al desarrollo humano, social y económico del Perú y del pueblo peruano, teniendo como primer destinatario el entorno circundante, es decir, el Cono Norte.

¿Qué recibe, por lo tanto, el estudiante que se matricula en la UCSS?

Primero, recibe una formación académica de alta calidad, que contribuye al crecimiento de profesionales responsables y preparados. Sin embargo, el valor añadido de la oferta de la UCSS se encuentra en lo que ya hemos subrayado, es decir, en la atención al estudiante como individuo y, por lo tanto, en el propósito formatvo a nivel humano. La excelencia en la docencia no son el objetivo por conseguir, sino una condicio sine qua non, el punto de partida imprescindible sobre el cual construir el posicionamiento estratégico e intentat conseguir una ventaja 


\section{Riccardo Spinelli}

competitiva. Lo que la UCSS se plantea es ofrecer al estudiante mucho más que un simple curso de formación: la organización se enfoca en el estudiante y lo considera, además de «cliente», hasta ktecurso», de cierta manera parte del activo patrimonial; los estudiantes son considerados la verdadera riqueza de la Universidad.

Segundo, la UCSS se compromete en garantizar al estudiante un ambiente de estudio serio, y al mismo tiempo sereno y acogedor. Este aspecto es de gran importancia, sobre todo en un contexto social como aquel del Cono Norte, donde sentirse seguros y tutelados es una condición absolutamente no común. En la estructura organizativa existe un sector apropiado, denominado «Servicios de Bienestar Estudiantiby, que se ocupa de todas las iniciativas en apoyo de la calidad de vida de los estudiantes: asesoria psicológica, orientación acadétnica y al trabajo, seguro médico y servicio de primeros auxilios, organización de eventos deportivos, entrega de becas de estudio. También en esto se nota la distinción entre la UCSS y las otras universidades; no se quiere crear una estructura eficiente pera despersonalizada, sino una verdadera comunidad de estudio en la cual el joven pueda expresar sus potencialidades y relacionarse de manera proficua con los demás. Con respecto a eso, es emblemático un comentario, sólo aparentemente banal, muy común entre los gerentes; «La Universidad funciona bien si los estudiantes están contentosw.

Pasando a la tercera modalidad de acción, es oportuno evidenciar las muchas iniciativas de formación cultural y espiritual organizadas con frecuencia: seminarios, charlas, encuentros, cursos de formación, etc. Se intenta favorecer el crecimiento del individuo en su entereza, prestando la debida atención a la esfera espiritual, como propia de cualquier institución que se define wcatólica».

Quedan por analizar las actividades de producción de bienes y servicios en sentido estrecho. Algunas, como el comedor, los talleres informáticos, la producción de material didáctica o la biblioteca, funcionan sobre todo para los estudiantes, mientras el CEASE, el Centro de Idiomas y el Centro Preunivetsitario tienen una clientela potencial incluso, o sobre todo, externa a la UCSS.

\subsection{El sistema compecitivo}

Se puede analizar el sistema competitivo en el cual actúa la UCSS por medio del modelo de las cinco fuerzas competitivas de Porter. Sin embargo, es una premisa fundamental evidenciar que la relación con las otras universidades o

'PORTER M, 1979, sHlow competitive forces shappe strategyn en Harnard Busimess Rmien, marzoabril; PORTER M, 1999, Ilewioggio competiave. E.diciona di Comunita 


\section{La Universidad como empresa non profit: el casa de Ta Universidad Católica Sedes Sapientiae.}

instituciones similares no tiene que ser siempre y solamente competitiva, porque el tapo de actividad 5 el gran numero de usuarios potenciales (varios miles de estudiantes sólo) en Lima hacen posible la creación de un sistema de colaboración para perseguir los comunes objetivos de apoyo al desarrollo del país".

Dicho eso, el sector en que se encuentra la UCSS parece escasamente competitivo, por lo menos en el corto plazo.

A pesar de que Lima tiene más de veinte universidades, razones económicas (c) nivel de las pensiones), geográficas (la gran distancia entre el Cono Norte y el centro de la ciudad) y académicas (excesiva dificultad de los exámenes de admisión) limitan mucho el número de efectivos competidores. Los jóvenes del Cono Norte, residentes en barrios periféricos, con baja capacidad reditual y procedentes de colegios de baja calidad, tienen escasas oportunidades de acceso a la educación universitaria; pero la UCSS, gracias a su politica de perisiones bajas (las más bajas de Lima) y a li proxirnidad geografica, se pone como alternativa posible y sobre todo de alta calidad.

Además, estas caracteristicas socioeconómicas del segmento principal de clientela lo hacen muy poco actractivo para nuevos sujetos económicos que decidan entrar en el business (potenciales nuevos entrantes), ya desalentados por los altos costes necesarios para poner en marcha una simil empresa.

La oferta de servicios alternativos a la formaciôn universitaria está constituida principalmente por cursos de formación técnico-profesional o por la entrada en el mundo del trabajo: con respecto a lá primera tipologia, la Universidad coopera activamente con un instituto de formación patrocinado por la Diócesis de Carabayllo; mientras es la misma UCSS, a través del CEASE, la que promueve iniciativas de apoya a la empresarialidad juvenil. No se trata, de hecho, de verdaderos substitutos del eproducto universidads, porque tienen en común un mucho más temible senemigon: el kno hacer nadas.

En efecto, la prioridad que una entidad como la UCSS se propone es, más que crecimiento del número de sus estudiantes, la disminución de la cantidad de jóvenes que pasan su vida desamparados en la calle, sin trabajo, y vuelven muy pronto a ser presa de la criminalidad.

Con respectò, luego, a la relación con los proveedores (poder contractual de los proveedores), se quiere proponer una interpretación original de este aspecte competitivo. Más que con los proveedores atradicionales» de bienes y servicios (energia eléctrica, gas, agua, articulos de escritorio, etc), hacia los cuales la

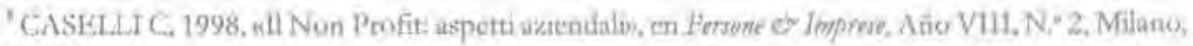
UMES.
} 


\section{Riccardo Spinel1i}

Univers:dad tene una buena uposición de fuerzas gracias a su dimensión cconómica en el entorno del Cono Norte, es interesante analizar la relación con el verdadero imput del proceso de producción, la umateria priman que etransforman: el estudiante que acaba de egresar del colegio. Por eso es evidente la necesidad de establecer profundos vínculos con las escuelas secundarias, para dirigir hacia la UCSS a los estudiantes que cada año egresan de la secundaria. Para conseguir este resultado, son muy oportunas las actividades de presentación y orientación que la UCSS hace en todos los centros de enseñanza secundaria en el Cono Norte y el establecimiento de canales privilegiados de diálogo y cooperación con las escuelas católicas, en particular aquéllas que gravitan alrededor de las parroquias y de la Diócesis. Hay que administrar muy bien la imagen y la reputación de la institución, utilizando todos los canales de propaganda y, sobre todo, dejando que los resultados concretos actúen como atractivo para los potenciales nuevos estudiantes.

Análogamente, con respecto al estudiante como uclientes (poder contractual de los clientes), es admirable la atención a su satisfacción, de manera que se reduzcan cuanto más los casos de abandono y de cambio de Universidad. No se puede olvidar que un cliente insatisfecho, aun obligado por las circunstancias a no cambiar proveedor, puede tener un efecto muy negativo sobre la erogación del servicio y sobre la imagen y reputación de la organización, haciendo propaganda negativa entre los potenciales nuevos clientes: las consecuencias de un comportamiento simil pueden ser mucho peores de las que son causadas por la pérdida de un singulo cliente. Desde este punto de vista, la presencia de altos vavitching costs para cambiar la Universidad puede ser la típica karma de doble filon, porque deciene en la órbita de la empresa un estudiante insatisfecho y, consecuentemente, un potencial elemento destabilizador.

\subsection{El sistema de los actores sociales y la propuesta proyectual}

Los siguientes dos puntos del modelo están muy profundamente conectados entre si, porque la empresa ofrece a cada típologia de intelocutor una propuesta diferente y espera diferentes formas de contribución y consenso.

Porlo tanto, es conveniente analizar individualmente los grupos a los cuales la UCSS se dirige, intentando delinear cada vez las líneas guia de la propuesta proyectual.

Se pueden identificar, en el entorino social relacionado con la UCSS, las sigurentes categorias:

- empleados

- financiadores 


\section{La Universidad como empresa non profit: el caso de la Universidad Católica Sedes Sapientiae.}

\section{instituciones y colectividad local}

La erapresa tiene que instaurar un diálogo proficuo con todos sus interlocutores, porque el conseguimiento del los objetivos estratégicos está usociado indisolublemente con el nivel de consenso ${ }^{\top}$. Se busca el conseguimiento de tal consenso con la persuasión, es decir, la confrontación crítica sobre el proyecto estratégico. Es una opción que empeña mucho, a la cual hay que destinar muchos recursos, sobre todo en un sentido humano y relacional; sin embargo, es aquella que asegura los resultados más válidos y duraderos, con óptimas repercusiones sobre la eficiencia y la eficacia de los procesos empresariales; además, es muy coherente con los valores (la colaboración, el diálogo, la transparencia,) que están a la base de las organizaciones sin afán de lucro, y eso hace que su aplicacion sea aun más auspiciable.

Examinemos ahora cómo la UCSS administra su relación con las diversas categorias sociales de referencia.

En las empresa de servicios, caracterizadas por una fuerte personality intensity", la relación con los empleados es fundamental. En el caso de la UCSS, su aspecto más importante es sin duda la necesidad de asegurar, dentro de la organización, un elevado nivel de uniformidad por lo que concierne a los valores y la cultura: la visión institucional tiene que penetrar en toda la estructura, de manera que el cliente la perciba plenamente.

Esta necesidad de cohesión representa seguramente una criticidad del proceso de desarrollo de la Universidad, sobre todo teniendo en cuenta su próximo crecimiento dimensional, que solicirará un incremento de los recursos humanos. Los métodos tradicionales de selección pueden ser inadecuados, porque es muy difícil coger un aspecto tan intangible como la presencia de uria visión compartida. Adquiere, por lo tanto mucha importancia el contacto personal con el candidato, por ejemplo, a través de una entrevista.

La persornel idea " que Ia UCSS propone está centrada en la figura de un empleado joven, muy motivado y deseoso de aprender, más bien próximo, por cultura y valores, a la visión institucional. El perfil rerributivo estí a un nivel parecido a aquél de las otras universidades de Lima, porque no se puede ignotar la importancia del factor económico si se quiere contratar profesionales de alta calidad; por otro lado, las peculiaridades de la UCSS, la fuerte proyección social y la ausencia de finalidad lucrativa pueden constiour factores de arracción para

\footnotetext{
CODA V., 1988.

'NORMANN R., 7996, Le gestione strategiax des senvin, Miluno, ETASI.IRRI

'Tbiders.
} 


\section{Riccardo Spinelli}

algunos segmentos de la fuerza laboral, sobre todo en consideración de la siempre mayor importancia que se atribuye, en la evaluación de una oferta, a los elementos extraretributivos, primero de todos la calidad del ambiente de trabajo. Desde este punto de vista, la UCSS está muy bien posicionada, porque la misma atmósferá serena que los estudiantes parecen apreciar mucho se percibe incluso en las oficinas, lo que hace más agradable la ejecución de las tareas cotidianas.

El principio fundamental es la colaboración, como esfuerzo conjunto para el conseguimiento de una meta común; crecimiento de la Universidad y bienestar del individuo no son objetivos antitéticos, al contrario, hay que verlos como una cosa sola, porque el éxito de la empresa está ligado estrechamente con el grado de desarrollo social que logra favorecer, y los empleados son uno de los interlocutores sociales más relevantes.

De los empleados, la UCSS espera en primer lugar empeño y cooperación, y lo más importante, también una implicación no exclusivamente profesional sino personal, para que sientan ser parte de un gran proyecto de desarrollo, dirigido precisamente a aquellas realidades sociales y económicas desde las cuales ellos mismos provienen a menudo. Si esta comunión de propósitos es el valor añadido pedido, ella representa también el valor añadido ofrecido, porque la UCSS se propone como el instrumento más idóneo para que las potencialidades y capacidades del empleado den el mejor resultado en el perseguimiento de una finalidad que él siente propia y comparte.

Viniendo ahora a la politica de gestión de los financiadores, hay que subrayar su importancia fundamental en la UCSS como en todas las empresas non profit.

La UCSS tiene una gran ventaja porque, por ella, la Diócesis de Carabayllo no es sólo un financiador sino el promotor, papel mucho más ncomprometedon; por eso no se necesita realizar actividades de fund raising dirigidas a la Diocesis, dado que ella está ya formalmente comprometida en el apoyo a la íniciativa educativa. De todas maneras, uno de los objetivos estratégicos de la UCSS es precisamente soltarse de la dependencia financiera y lograr autofinanciar las actividades corrientes con sus rentas. Sin embargo, hay que evidenciar cómo la Diócesis segirá siendo, incluso en la visión de largo plazo, la fuente de los recursos necesarios para las grandes inversiones extraordinarias, pot ejemplo, una ampliación infraestructural.

\footnotetext{
"KOTLER R., ANDREASEN A., 1988, Marketing per le organizazioni non profiti la grande saita

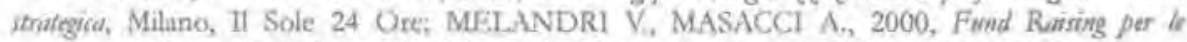

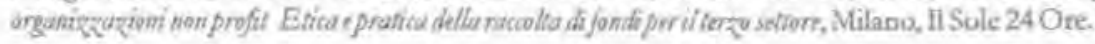




\section{La Dniversidad como empresa non profit: el caso de la Universidad Católica Sedes Sapientiae.}

Con respecto a los otros aportadores de capital, un pequeño grupo de entidades públicas y privadas tiene un papel de primaria importancia. A la hora de empezar la realización de su usuenoo», Mons. Panizza ha recibido la concreta ayuda de unas personas a él próximas y, en misura menor, de administraciones locales y universidades italianas, gracias a proyectos coordinados por algunas ONGs de varias nacionalidades. Para edificar un proficuo diálogo con sujetos de este tipo, es fundamental demostrar mucha seriedad y competencia, puesto que estas envidades solicitan una documentación muy detallada sobre las iniciativas que son llamadas a financiar; por otro lado, son seguramente, entre los potenciales financiadores, aquellos más sensibles al impacto social de las propuestas, ya que su atención está fuertemente concentrada en el interés público. Por lo tanto, se pone particular atención en la organización de los encuentros con los representantes de las entidades interlocutoras: en el tiempo limitado a su disposición, los miembros de las asi llamadas «misiones explorativas», deben lograr coger los rasgos distintivos de las actividades de la organización que pide el firianciamiento. Es muy positivo que la UCSS comparta con los principales financiadores las lineas de intervención que están en la base de su misión: son relevantes, en este propósito, la gran importancia que se da, incluso en los programas de la Unión Europea, al desarrollo de la empresarialidad juvenil, imbito en el cual el CEASE puede ser catalizador de intereses y fondos, y la prionidad que inuchas ONGs dan a los proyectos de formación y educación.

Se cree oportuno concentrar el flujo de inversiones principalmente en aquellas actividades en que se desempera más la proyección social de la Universidad, porque el core business didáctico, además de ser dificilmente financiable por los sujetos mencionados, es capaz, por lo menos en la visión estratégica de largo plazo, de autosustentarse.

Al contrario, tienen una importancia marginal las donaciones individuales, forma de cooperación para la cual se espera un crecimiento. Una posible forma de incitación podría ser un sistema de ayuda a distancia, en el cual un donador se hace cargo, con pagos periódicos, de las pensiones de un estudiante con dificultades económicas.

Otro grupo de potenciales financiadores que es muy poco activo está constituido por las empresas, peruanas y extranjeras, activas en Lima. Sin embargo, se está organizando un sistema de pasantias: una forma de ¿financiamento indirecto" para aquellos estudiantes que están próximos al final de la carrera y, por lo tanto, a la entrada en el mundo del trabajo; de esta manera se ofrecería a los jóvenes una oportunidad para acercarse a la realidad laboral y, al mismo tiempo, incrementar su renta, mientras las empresas tendrian por un lado 


\section{Riccardo Spinelli}

un muy buen retorno de imagen $y$, por otro, el acceso a recursos humanos calificados, eventualmente integrables en su plantilla al final de la pasantia. Para las ermpresas sería incluso una forma de lo que Porter y Kramer ${ }^{\mathrm{t}}$ llaman context-focused philanibropy o "filantropía estratégica», es decir, una intervención de ayuda enfocada en el entorno estratégico con una convergencia de intereses entre el beneficio social para la colectividad y aquello económico para si mismas, detivante del desarrolo del entorno de referencia.

Desce un punto de vista estratégico, en efecto, la filantropia puede ser una manera muy eficiente para mejorar el contexto competitivo, porque coordinándose - como en el caso de la UCSS - con instituciones non profit, las empresas pueden utilizar con más eficacia sus competencias y tecursos para favorecer el cambio a nivel socioeconómico.

Con respecto, finalmente, a la relación con las instituciones y con la colectividad local, es importante la necesidad de tutelat y desarrollar hacia el exterior la imagen de la Universidad, sin duda uno de sus "activos" de mayor valor. En este marco se colocan la organización de frecuentes eventos, para tener una presencia constante en el panorama cultural y acadérnico de la ciudad, y la publicación de Studium, una revista semestral que reúne artículos de eminentes personalidades académicas sobre temas culturales de gran relieve y acerca de los resulados de las investigaciones hechas en la UCSS.

La UCSS intenta siempre presentarse a las instituciones públicas de la mejor manera posible, sobre todo con aquellas que son más próximas a su área de actividad, por ejemplo, el CONAFU, que cada sño, por los primeros cinco años, tiene que evaluar su gestión y autorizar la prosecución de las actividades. $\mathrm{La}$ importancia de las relaciones públicas es ran grande que justifica el gran empeño de recursos humanos y financieros y la presencia de una oficina dedicada, la Oficina de Promoción e Imagen Institucional.

\subsection{La estructura}

Se intenterá ahora analizar la estructura de la UCSS en un sentido muy amplio, que incluye tro sólo la organización y los mecanismos operativos, sina también todos los recursos, humanos y no, que constituyen el patrimonio tecnalógico, comercial, directivo y económico-financiero de la empresa.

\footnotetext{
"PORTER M., KRAMER M., 2002, wThe cumpetitive advantige of corporate philanthropys en Hartiard Business Review, Dicembre

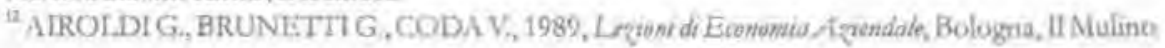




\section{La Universidad como empresa non profit: el caso de la Universidad Catōlica Sedes Sapientiae.}

Con respecto a la infraestructura, está claro ya ahora que cuando las carreras sean completamente activadas con todos los ciclos en marcha, la sede actual no sera suficiente para todos los estudiantes. Las alternativas que los gerentes están examinando prevén o la ampliación del edificio actual (ya en obras) o el traslado en un nuevo inmueble, construido ex novo en una zona adyacente.

Por lo que atañe al diseño de la organización de la UCSS, se necesita una premisa sobre la autonomia administrativa de la Universidad.

Como ya se ha evidenciado, la ley peruana obliga a las universidades recién creadas a subyacer por los primeros cinco años al control de una entidad pública, el CONAFU. Las consecuencias operativas son muchas: en primer lugat, los administradores tienen que entregar una rendicontación anual de gestión al CONAFU, cuya aprobación es indispensable para poder seguir funcionando; segundo, el Estatuto y los reglamentos internos del ateneo son provisionales y tendrán que ser aprobados por el CONAFU; tercero, los órganos de gobiemo de la Universidad no tienen los plenos poderes y su denominación es temporal.

A pesar de esta situación de inestabilidad, se puede analizar la organización interna de la UCSS con los modelos clásicos de la teoría, y el más apropiado parece ser el fincional, en el cual ka los órganos directivos de primer nivel se otorga la responsabilidad de grupos de procesos afines en un sentido técnico y económicon ${ }^{13}$.

La actividad de la UCSS se divide, en efecto, sea a nivel operativo sea organizativo, en dos áreas funcionales, una administrativa y otra académica; la dirección general pertenece a un órgano colegial, la "Comisión Organizadora y de Gobiernon, presidida por el Presidente, mientras cada área funcional tiene un Vicepresidenté. En el caso en análisis, la estructura funcional parece la más idónea, porque la heterogeneidad de las dos áreas de trabajo y la peculiaridad de los uprocesos productivos llevan a la necesidad de separar claramente entre el ámbito administrativo y el académico; se requieren competencias profesionales y estilo de management muy diferentes, puesto que la didáctica tiene un nivel de persanality intensity seguramente mayor que la gestión contable y administrativa. La confirmación de esta hipótesis viene de la constatación de la criticidad que surge en la frontera entre las dos áreas, con respecto a aquellos profesionales que, aun operando a nivel administrativo, tienen frecuentes contactos con el público.

Los órganos de la UCSS que corresponden a la uproducción de bienes y serviciosn, en particular el Centro Preuniversitario, el CEASE y el Centro de

\footnotetext{
"PAOLETTI F. 1997, da strutura elencntare e funzionales en COSTA G., NACAMLILI R. C.

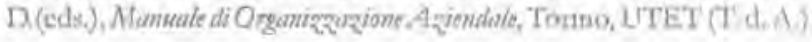




\section{Riccardo Spinelli}

Idiomas, actualmente constituyen parte integrante de la estructura de la UCSS y sus responsables dependen directamente de la Comisión Organizadora; los tres centros no son adminis trativamente autónomos y deben adecuar su actividades a las necesidades, sobre todo de espacios, de la actividad académica. Esto provoca fuertes repercusiones operativas, y dado que las tres instituciones generan constantes flujos de renta y buenos beneficios, es probable que cuando sea posible se les dará más independencia. En el diseño final serian centros satélites de la Universidad, con la cual se coordinarian y a la cual destinarian en la parte no reinvertida sus beneficios; dentro del proceso de desarrollo de la UCSS, podrian tener una sede propia, que gestarian en total autonomia. El primer paso, de todas maneras, deberia ser la decisión de financiarlos con un budget y no con la cancelación de los síngulos gastos, para responsabilizar el management y conseguir un mayor nivel de eficiencia y eficacia. Además, no se puede olvidar que las caracteristicas del business de los tres centros, en particular del centro de idiomas, hace que produzcan un constante flujo de renta, muy útil para regularizar el casbflow total de la Universidad, acondicionado por la concentración de las entradas en el periodo de la matrícula y del pago de las pensiones.

El último aspecto que queremos examinar concierne a las relaciones institucionales con otras organizaciones afines, en el Perú y en el exterior.

Desde siempre, la UCSS se ha caracterizado por la fuerte protección internacional, que se ha concretado en muchos acuerdos que la asocian, directamente o a través del CEASE, con numerosas universidades: entre ellas se encuentran l'Univetsità di Genova (Italia), I'Universita Cattolica del Sacro Cuore di Milano (Italia), la Universidad de Alicante (España), la Universidad Particular Anteno=Orrego de Trujillo (Perú) y el ESAN (Perú).

Con estas convenciones se busca conseguir formas de colaboración académica en diferentes áreas: intercambio de estudiantes y profesores, cooperación en proyectos de investigación, condivisión de know-bow. En un mundo que se define no siempre correctamente más y más uglobalizadon, es muy oportuno seguir reforzando esta red de relaciones entre la UCSS y otros ateneos, para conseguir con la misma terminologia la uglobalización de la cultura y de la solidaridadr. Acercar estudiantes y profesores de otros paises a un contexto como el peruano puede llevar, en efecto, a la maduración de una conciente atención hacia los problemas de los paises en vía de desarrollo.

\section{Conclusiones} la UCSS?

¿Cuáles son, en conclusión, los rasgos principales de la fórmula empresarial de 


\section{La Universidad como entpresa non proftt: el caso de la Universidad Católica Sedes Sapientiae.}

Para evaluarlos es ítil leer las cinco variables del modelo como kun sistema dinámico unitarion ${ }^{1+4}$, en el cual se influencian recíprocamente y contribuyen a la determinación de una resultante final.

Comentando los resultados obtenidos, hay que subrayar un aspecto muy positivo, es decir, la gran consonancia entre los elementos de la estrategia de la UCSS. La consonancia es sinónimo de coherencia, sintonia y armonía, y es uno de los elementos que más influyen sobre el grado de éxito de una estrategia: en las ermpresas exitosas atodos los elementos de la fórmula competitiva... representan... el desarrollo lógico de un idea central sobre la manera de ser competitivos en aquel particular sector económico» ${ }^{15}$.

En el caso de la UCSS, la idea central es presentarse al mercado con una oferta de al ta calidad que, sin embargo, por el hecho de no tener finalidad luctativa, puede ser propuesta con un precio bajo. Consecuentemente es posible conquistar un segmento de mercado bastante seguro, puesto que se refiere a un entorno territorial y económico del Cono Norte donde la competencia es minima y presenta muy poca atractividad para potenciales nuevos entrantes. La fuerte proyección social de la UCSS hace que sea muy bien aceptada en el panorama social y eso facilita, por lo menos en parte, la búsqueda de los recursos necesarios para la sobrevivencia y el crecimiento de la institución. La estructura está dimensionada según las actuales disponibilidades económicas, pero con la mirada siempre hacia adelante.

Hasta ahora la gestión de la Universidad ha sido muy atenta y competente, además de hábil en disfrutar las fortalezas de la organización. Sin embargo, ecuáles son estas fortalezas?

La primeta es la fortisima coherencia interior, que se cancreta, como ya hemos acordado varias veces, en el hecho de que la visión y la misión instutucionales son aceptadas y perceptibles en toda la estructura. En segundo lugar, desde un punto de vista estrictamente competitivo, el posicionamiento de la UCSS es estable y bastante seguro, capaz de asegurarle por lo menos unos años de competencia baja; además, el gran número de potenciales estudiantes permite mirat al mañana con optimismo, dado que el mercado, por el lado de la demanda, parece en constante crecimiento. En tercer lugar, la Universidad ha conquistado, a pesar de que funcione desde menos de tres años, una óptima reputación en el ambiente académico, científico, cultural y económico del Perú; esto es muy importante para una institución que, a nivel global, se mueve hacia una estructura de red, smàs y

\footnotetext{
${ }^{3 *} \mathrm{COD} N \mathrm{\gamma}, 1988,(1 \mathrm{~d}, \lambda)$

"Ibidem.
} 


\section{Riccardo Spinelli}

más conectada con otras grandes organizaciones, con otras universidades, con sus mismos clientes y co-maker a través de relaciones culturales y conexiones telemáticas $\%^{16}$. A este aspecto se reconduce otra fortaleza de la UCSS, su densa red de relaciones internacionales. Última en orden, pero no por importancia, se evidencia la buena solidez económica y financiera, sobre todo en el contexto de los planes de desarrollo estratégico de la Universidad.

Por supuesto, la UCSS presenta también algunas debilidades, sea en la estructura sea en la organización.

Además de la limitada disponibilidad financiera, que condiciona muchos aspectos estratégicos, la carencia peor es-en mi opinión- de cmentalidad empresariabs. No se ha difundido todaviá completamente una impostación de la Universidad como empresa: la actividad educativa es considerada como un área en la que poca importancia deben tener las razones económicas, sacrificadas a las superiores finalidades morales y culturales.

Esta visión es - a mi parecer-errada o por lo menos incompleta, porque un acercamiento alcance «técnicon al problema gestional no disminuye ni desvaloriza los contenidos, sino hace posible una mayor eficiencia y eficacia. La finalidad institucional de la UCSS es muy ambiciosa: llevar la enseñanza universitaria al Cono Norte es un reto que más que una persona ha definido ximposiblen. Si se quiere conseguir hay que darse cuenta de la necesidad de un aposte cientifico, técnico y managerial.

Con respecto a eso, es muy positivo -en mi opirión-que los gerentes de la Universidad, después de un primer período creativo en el cual casi todos eran especialistas de pedagogia y filosofia, sean ahora en mayor número docentes de ciencias económicas y, en particular, de ciencias de la administración, porque con su contribución estas personas favorecerain significativamente al conseguimento de metas más y más ambiciosas.

A pesar de estas debilidades, el nivel de satisfacción de los usuarios, los estudiantes, es bastante alto; soportan esta afurmación los resultados de un detallado cuestionario que hice, con una muestra de 200 estudiantes, durante mi estancis en Lima, en el verano del año 2001: en una escala de 1 (minimo) a 5 (máximo), la evaluación global del servicio ha sido de 3,7 ; con el $90 \%$ de los entrevistados que afirma estar usatisfechon. Los estudiantes parecen apreciar mucho el clima sereno y humano que se respira en la Universidad, tanto que lo consideran uno de los aspectos en los cuales más fuerte es la conotación católica. Es también digno de atención el hecho de que los entrevistados, aun admitiendo

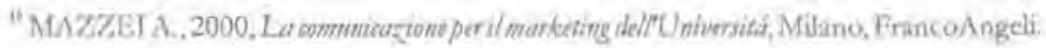




\section{La Universidad como empresa non profit: el caso de la Universidad Católica Sedes Sapientiae.}

haber elegido la UCSS sobre todo por el nivel bajo de las pensiones, ahora la aconsejarian a potenciales nuevos estudiantes, no tanto por la economicidad, sino por el nivel alto de la docencia y, en general, de la organización: esto significa que durante su experiencia universitaria han sido satisfechos por el servicio recibido, cuyo nivel es a menudo muy superior a sus expectativas.

\subsection{El problema de la reproducibilidad}

Sin embargo, ¿se puede reproducir la exitosa formula empresarial de la UCSS en otros contextos de subdesarrollo?

Dada por descontada la importancia de los elementos contingentes y la consecuente imposibilidad de una perfecta reproducibilidad, pienso que la UCSS puede representar un rexperimentom exitoso y repetible. Su naturaleza de empresa non profit permite mirar a los comunes indicadores económico-financieros con algo de elasticidad, pero al mismo tiempo impone una gestión atenta y prudente de los recursos; en los paises del Tercer Mundo, inestables y de dificil alecturay, estos son dos aspectos muy relevantes, porque por un lado surge de la necesidad de whacer un beneficion tipica del for profit; pero, por el otro, no deja espacio a la (improvisacióms o a una actitud de meta esobrevivencias. El ser empresa obliga al conseguimiento de la autonomia o por lo menos de la economicidad de la gestión.

De todas maneras, hay otros elemeotos que pueden constituir un obstáculo a la realización de iniciativas afinas a la UCSS.

En primer Iugar, se necesita una entidad promotora, como la Diócesis de Carabayllo en este caso, que se haga cargo de los gastos e inversiones de la fase de

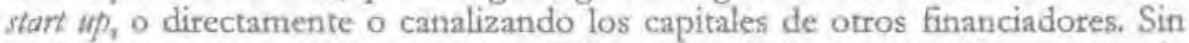
embargo, es otro cl factor aun más dificil de reproducir: el capital humano para la gestion del nuevo ateneo. Es innegable que una buena parte del éxito de la UCSS depende del esfuerzo y del empeño de quien le dedica cada día su tempo, consideríndolo mucho más que un trabajo; además, no se necesitan solamente capacidades técnicas, sino un impulso humano, una disponibilidad y una vocación al servicio muy fuera del ordinario. Hallar símiles (crecursos» es muy dificil, y aun más dificil es que estas personas, ya tan raras, se encuentren en un grupo y entren en contacto con un potencial financiador. En esta búsqueda, las organizaciones que opetan en la cooperación para el desarrollo pueden terier un papel muy importante, actuando como trimites y punto de agregación para los componentes humanos sobrecitados. 


\section{Riccardo Spine11i}

\subsection{La contribuciónal desarrollo}

Nos queda una última pregunta a la cual intentar contestar: ¿cuál es la manera en que una iniciativa como la UCSS puede contribuir, por supuesto en medida proporcional a su alcance, al desarrollo socioeconómico del Perú y, en general, de un pais en via de desarrollo?

Teniendo en mente el esquema del «diamante nacionaly de Porter", se puede afirmar que la acción más eficaz se produce sin duda en la *creación) de «recursos humanos).

Las universidades pueden tener un papel fundamental en la creación de (activos de este tipo, porque sproducen conocimientom: cada joven licenciado lleva consigo un gran valor aniadido, sus conocimientos lo hacen un profesional capaz y responsable que puede contribuir activa y proficuamente al desarrollo económico de su país; representa, en la terminología de Porter, un factor de la producciôn kavanzadò y kespecífico». Además, aun una iniciativa a pequeña escala como la UCSS, puede crear eslabonamientos importantes: como Mons. Panizza ha intuido en su visión de la Universidad, para crear «buenos alumnos" se necesitan ubuenos maestrosn, y la uproduccións de la facultad de Ciencia de la Educación puede representar un elemento de innovación en este sentido. Con respecto, luego, a los estudiantes de Ciencias Económicas, su contribución tendrả dúplice forma: por un lado su educación hará de ellos buenos empleados y, por el otro, les dará los instrumentos para crear nuevas empresas, alimentando de esta manera el ciclo de creación de la riqueza con nuevas actividades productivas.

Otro ámbito, tal vez menos perceptible, en el cual una atenta política de soporte a la enseñanza universitaria piede tener buenos resultados, son las "condiciones de la demanda agregadas, a nivel cualitativo y cuantitativo. Formar profesionales capaces de conseguir buenos trabajos $y$, consecuentemente, rentas superiores a la subsistencia, leva al crecimiento de la aclase median, un grupo social fundamental en las dinámicas de ios sistemas económicos. El incremento de las rentas implica la mejoria de la calidad de la vida, una mayor seguridad e inevitablemente mayores consumos; a nivel agregado, esto puede soportar el conseguimiento de aquel nivel de demanda minima potencial que muchas veces inhibe la creación de nuevas industrias en los paises en via de desarrollo ${ }^{18}$.

\footnotetext{
"PORTER M. 1990, wThe competitive advantage of Nationsn en Hamurd Bwsiness Revitw, marzoabril: PORTER M. 1991, I/ wameggro sompetitue delle naziom, Milano, Arnoldo Mondadori Editore

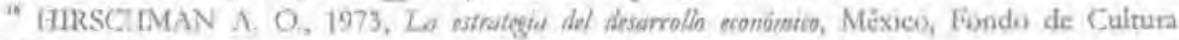
Económica.
} 


\section{La Universidad como empresa non profit: el caso de la Universidad Católica Sedes Sapientiae.}

Por último, es oportuno subrayar otro efecto de la actividad educativa de las universidades como la UCSS: la influencia sobre el "sistema de objetivos" de los individuos y de la sociedad. Es importante conocer los valores sociales que condicionan la actitud hacia el trabajo y se nota que a los jóvenes peruanos no les falta la voluntad de mejorar por sí mismos para contribuir al crecimiento de su pais, para que se libere de la condición de dependencia y subdesarrollo que to aptisiona $\mathrm{La}$ Universidad representa un medio de emancipación y realización personal que pueden ser proyectadas a nivel colectivo, Format una nueva generación, conciente y comprometida con el Perí, constituye un desafio cuyo éxito puede ser muy significativo.

Un papel fundamental para el conseguimiento de estos ambiciosos objetivos toca sin duda al gobierno, sin embargo, vale la pena precisar bien cuál será su efectiva área de competencia. En una visión liberal del Estado, no se requiere siempre y sólo una intervención directa en las actividades productivas: se le atribuye también el papel de crear los requisitos necesarios y acnuar como "árbitro» $y$ «revisotin ${ }^{1 !}$.

Iniciativas non profit como la UCSS se ponen como alternativas a ta intervención pública, a la cual piden la predisposición de las condiciones básicas para obrar: certeza del derecho, infraestructuras, seguridad, etc,

Es una opción estratégica fundada sobre la kcolaboración» y que puede llevar a grandes resultados: los gobiernos de los paises en vía de desarrollo empeñados en las batallas contra la pobreza, el hambre y las enfermedades de sus pueblos pueden encontrat en las entidades non profit valiosos aliados con los cuales cooperar para conseguir una mejor locación de los recursos ya tan escasos.

En conclusión, de la experiencia de la UCSS se puede sacat un mensaje de esperanza: podemos auspicar que, superando la dialéctica entre los modelos de desarrollo guiados por los gobiernos y los que son dejados a las espontáneas dinámicas del mercado, esta etercera vian fundada sobre la colaboración resulte ser la mejor forma para acompañar y apoyar a los países del Tercer Mundo en el camino largo y dificil del crecimiento, para que finalmente se afirmen a nivel global el bienestary la prosperidad.

\footnotetext{
"VITTADINI G., 1997, al cosa serve il nun profit: piú sucietă meno Statun en VTTTADINI G. (ed.), I/ non profit dimesato, Milano, ETASL.IBRI.
} 